\title{
Das Konzept der fluiden Bibliothek an der USB Köln
}

\section{Ralf Depping / USB Köln}

InetBib-ODOK Wien / 21. Feb. 2018 


\section{Ausgangslage USB Köln}

$>$ Magazinbibliothek / Numerus-Currens-Aufstellung

$>$ Archivbibliothek (ehem. SSGs, alte Stadtbibliothek, Sondersammlungen usw.)

$>$ hoher Anteil ungenutzter Bestände

$>$ i.d.R. immer noch wachsende Printbestände

$>$ steigender Flächenbedarf für Benutzerarbeitsplätze

$>$ denkmalgeschütztes Gebäude ohne Erweiterungsmöglichkeiten

$>$ Rara-Magazin in Planung 


\section{Bestandsmanagement}

$>$ Jede Hochschulbibliothek hat einen nicht unerheblichen Anteil an Print-Beständen, die aktuell nicht genutzt werden, aber aus guten Gründen nicht ausgesondert werden.

> Besucherlnnen der Bibliothek nutzen Bibliothek oft als Lernund Arbeitsort ohne Nutzung der Print-Bestände.

> Virtuelle Bestandspräsentation mit optimierten Portalfunktionalitäten ersetzt physische Bestandpräsentation.

> Ausweichmagazine preiswert „auf der grünen Wiese“ / auch Freihandbibliotheken gehen verstärkt dazu über, Bestände zu magazinieren. 


\section{Ausweichmagazin}

> Aktuell: konventionelles AWM mit ca. 800.000 Bänden Kapazität

> Projektiert: Vollautomatisches Hochregallager (ASRS) mit ca. 4.000.000 Bänden Kapazität $\rightarrow$ chaotische Lagerhaltung,, Aufbewahrung der Medien in Wannen, Ware-zur-PersonPrinzip mit Kommissionierungsstationen, sauerstoff-reduziert, erweiterbar und skalierbar 


\section{Ausweichmagazin}

\section{Vorbild: Speicherbibliothek Büron (Schweiz) dort z.Zt. 3 Mio. Bände Kapazität}

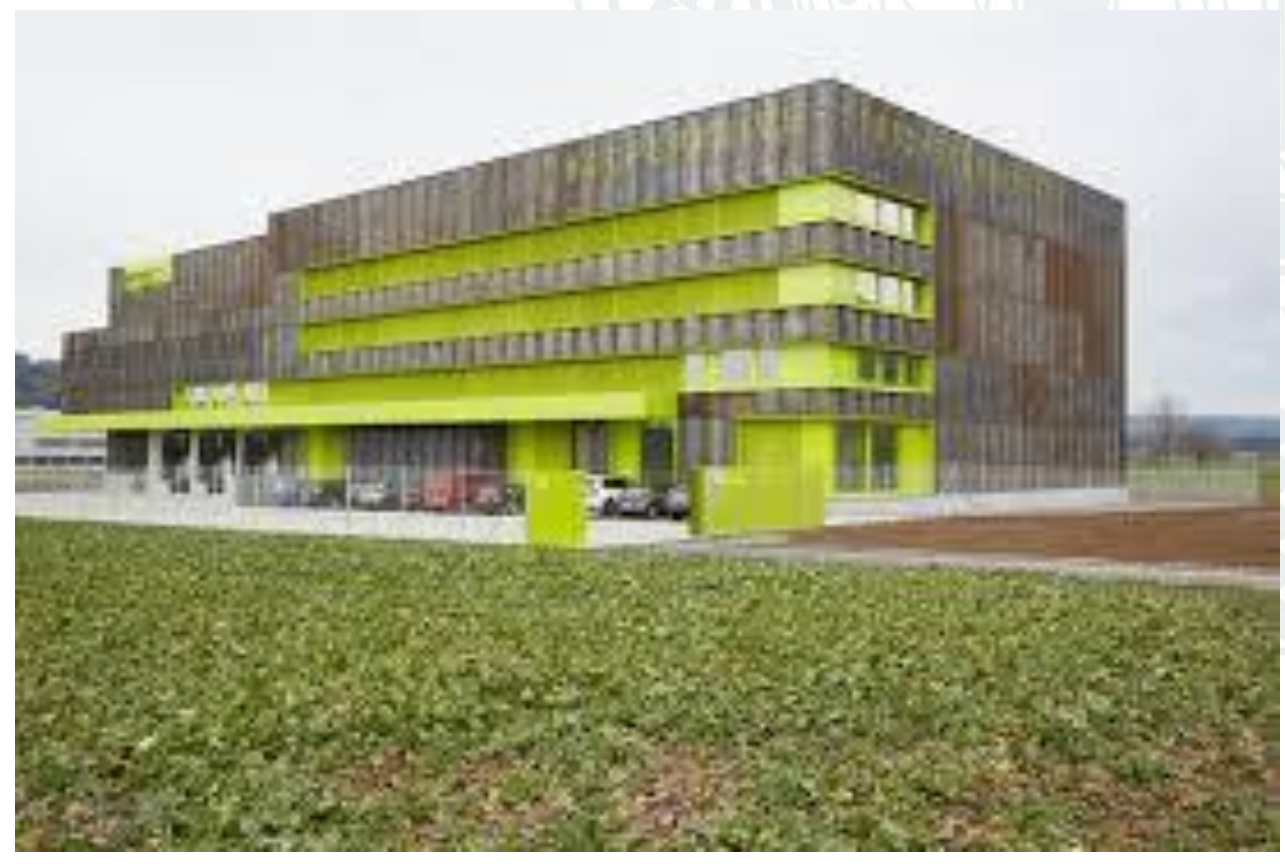




\section{Grundlagenuntersuchung}

\section{Ausweichmagazin}

$>$ Fachplaner: April-November 2017

$>$ Variantenentwicklung (manuell vs. vollautomatisch)

$>$ Konkrete Bedarfskalkulation (Grundstück, Gebäude) und Budgetierung (Investitionsund Betriebskosten) je Variante

$>$ Empfehlung ASRS-Variante

$\rightarrow$ Grundstückssuche in 2018 


\section{Chaotische Lagerhaltung}

> Ursprünglich Konzept aus der Lagerlogistik, nicht für Bereiche, die für Kunden zugänglich sind.

$>$ ASRS-Technologie (automated storage and retrieval system) $=$ vollautomatische Einlagerung und Bereitstellung, (z.B. UB Rotterdam) $=>$ u.U. sehr kapitalintensiv, aber Industriestandards, keine bibliotheksspezifischen Lösungen 


\section{Ausweichmagazin: Herausforderungen für Bestandsmanagement und -präsentation}

$>$ Aufwand für (Transport-)Logistik (geplant: $>90 \%$ der Bestände im Ausweichmagazin).

$>$ Zuordnung der Beständen zum Freihand- oder Magazinbestand (ggf. differenziert nach Magazin vor Ort u. Ausweichmagazin) nach Nutzungszahlen incl. Präsenznutzung.

> Umstellung zwischen Standorten i.d.R. aufwändig, erfolgte bisher für ganze Signaturengruppen und wird de facto nie revidiert. 


\section{Fluide Bibliothek}

> Das statisch-bestandsorientierte Konzept einer Aufstellung nach bibliothekarischer Ordnung wird im Freihandbestand zu Gunsten einer dynamischen Aufstellung in chaotischer Lagerhaltung aufgegeben.

$>$ Jeder Band (außer klassische Lesesaalbestände, LBS u. Rara) hat einen Standort im (Ausweich-) Magazin und steht nach Bedarf temporär im Freihandbestand. 


\section{Smart-shelves-Technologie}

> Freihand-Regale sind mit RFID-Antennen ausgestattet und liefern Informationen über den aktuellen Standort und über jede Entnahme (=Nutzung) eines Medium.

$>$ Medien können von Besuchern und Mitarbeitern an einen beliebig freien Platz eingestellt werden (ggf. differenziert nach Größe der Medien). 


\section{Dynamisches}

\section{Bestandsmanagement}

$>$ Alle (nicht-vorgemerkten) Medien, die aus der Nutzung zurückkommen, gehen automatisch in den

Freihandbereich (außer Rara, LBS).

$>$ Neuerwerbungen gehen auf Entscheidung des

Fachreferates in den Freihandbereich.

$>$ Abräumlisten für die Freihandbestände mit der geringsten Nutzungsfrequenz in Abhängigkeit vom zur Verfügung stehenden Platz.

$>$ Aktueller Standort (= Regalbrett) wird im Bibliothekssystem angezeigt und kann auch vor Ort z.B. über Smartphone recherchiert werden. 


\section{Katalognachweis}

$>$ Alle Medien behalten ihre normale Signatur, die auch in den Verbundkatalogen usw. zu sehen sein wird. Der Katalognachweis bleibt stabil.

$>$ "Smart-Shelves“ Ilefern just-in-time aktuellen Standort der Freihandbestände (Regalbrett) in eine temporäre Datei (= Konkordanz Signatur und Regalbrett in den „Smart Shelves“ im Freihandbereich), auf die das Bibliotheksportal bei Recherchen zugreift. 


\section{Anzeige im Bibliotheksportal}

$>$ Buch steht im AWM, Rara-Mag.: Anzeige von Signatur plus Bestell-Button.

$>$ Buch steht in LS bzw. LBS: Anzeige von Signatur ohne Bestell-Button.

$>$ Buch steht in Smart Shelves im FHM in chaotischer Lagerhaltung: Anzeige von temporärem Standort (Regalbrett, ggf. mit Zusatz „rechts“, „links“ oder „mitte") ohne Bestell-Button.

$>$ Buch aus FHM zur Präsenznutzung herausgenommen: ??? 


\section{Reduktion (Logistik-)aufwand}

> Alle intensiv genutzten Bestände im Freihandzugriff.

$>$ Zahl der Magazinbestellungen deutlich reduziert.

$>$ Umstellungen tagesaktuell möglich, ohne die Notwendigkeit von Katalogkorrektur.

$>$ Rückstellen ausgeliehener Medien an beliebigen Standort schnell und auch für Laien möglich.

$>$ Fehlertolerant: keine „verstellten“ Medien.

$>$ Permanente Inventur, permanente Messung der Präsenznutzung.

$>$ Aber natürlich auch extreme Abhängigkeit von der Technik (Ausfall-und Fehlersicherheit). 


\section{Aktueller Status des Projektes}

Angebot eines Firmenkonsortiums liegt vor

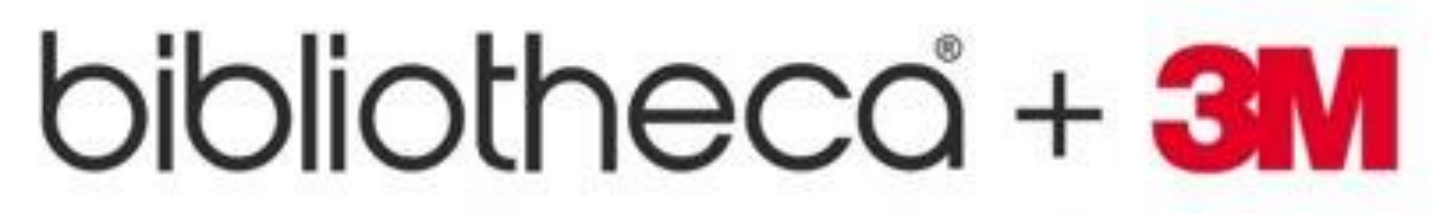
the best of both worlds
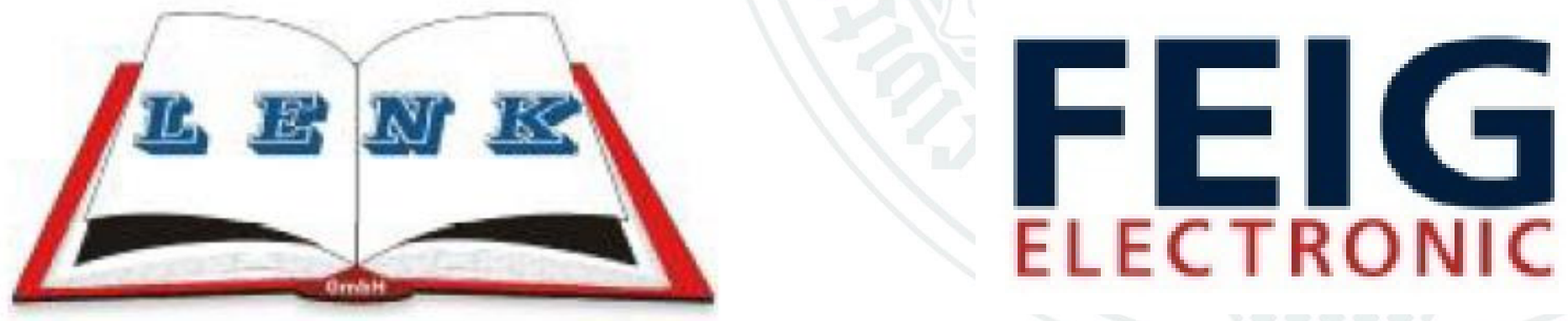

Finanzierung im Kontext des AWM-Neubaus projektiert. 


\section{Vielen Dank für Ihre Aufmerksamkeit}

